\title{
ON $L_{n}$ SETS, THE HAUSDORFF METRIC, AND CONNECTEDNESS
}

\section{A. M. BRUCKNER AND J. B. BRUCKNER}

1. Introduction. A set $S$ in the Euclidean plane $E_{2}$ is called an $L_{n}$ set provided any two points in $S$ can be joined by a polygonal line, of at most $n$ segments, lying entirely in $S$. Horn and Valentine [1] characterized such sets for the case $n=2$ and studied properties of the complements of such sets. It is clear that the notion of $L_{n}$ sets can be regarded as a generalization of the notion of convex sets and as a specialization of the notion of connected sets. Since polygonally connected sets are in some sense a limiting case of $L_{n}$ sets, it seems reasonable to expect that $L_{n}$ sets can be used to approximate polygonally connected sets. As we shall see in the sequel, any compact connected set (whether polygonally connected or not) can be approximated by compact $L_{n}$ sets. We will use the notation $\left\langle p_{1}, p_{2}, \cdots, p_{n+1}\right\rangle$ for the $n$-sided polygonal line ( $n$-line) joining $p_{1}$ to $p_{n+1}$ where $p_{2}, \cdots, p_{n}$ are consecutive, intermediate vertices.

2. The metric space $\Re$. In what follows, we will make use of a wellknown metric space whose elements are the compact sets of the plane.

Let $S$ be a compact set in $E_{2}$ and let $\epsilon>0$. The set

$$
S_{(\epsilon)} \equiv\left\{p \in E_{2}: \rho(p, S) \leqq \epsilon\right\}
$$

is called the $\epsilon$-parallel body of $S$. Here $\rho$ is the Euclidean metric of $E_{2}$.

TheOREM 1. The parallel body $S_{(e)}$ of an $L_{n}$ set $S$ is an $L_{n}$ set for the same $n$.

Proof. Let $x$ and $y$ be points of $S_{(\epsilon)}$. There exist points $x^{\prime}$ and $y^{\prime}$ in $S$ for which $\rho\left(x, x^{\prime}\right) \leqq \epsilon$ and $\rho\left(y, y^{\prime}\right) \leqq \epsilon$. Now $x^{\prime}$ and $y^{\prime}$ can be joined by an $n$-line $\left\langle x^{\prime}, p_{2}, \cdots, p_{n}, y^{\prime}\right\rangle$ lying in $S$. The segments $\left\langle x, p_{2}\right\rangle$ and $\left\langle p_{n}, y\right\rangle$ lie in the parallel body $\left\{\left\langle x^{\prime}, p_{2}, \cdots, p_{n}, y^{\prime}\right\rangle\right\}_{(e)}$ which in turn is contained in $S_{(\epsilon)}$. Therefore, $\left\langle x, p_{2}, \cdots, p_{n}, y\right\rangle$ is an $n$-line in $S_{(\epsilon)}$ joining $x$ and $y$.

Let $S$ and $T$ be compact sets in $E_{2}$. Let,

$$
d(S, T)=\inf \left\{\epsilon: T \subset S_{(\epsilon)} \text { and } S \subset T_{(\epsilon)}\right\} .
$$

Then $d$ is a metric on $\mathcal{K}$ the class of all compact sets in $E_{2}$. For a discussion of some of the properties of $\mathscr{K}$ see [2]. In particular, it is shown there that $\mathcal{K}$ is complete. Some additional properties of $\mathcal{K}$ are enumerated in

Received by the editors July 21, 1961 . 
Theorem 2. (a) If $S \in \Re$ then $S=\lim _{\epsilon \rightarrow 0} S_{(\epsilon)}$.

(b) If $S^{1}, S^{2}, \cdots$ is a decreasing sequence of sets, each a member of $\Re$, then

$$
\lim _{k \rightarrow \infty} S^{k}=\bigcap_{k=1}^{\infty} S^{k}
$$

(c) Let $n$ be a positive integer. The limit $S$ of a sequence $\left\{S^{k}\right\}$ of compact $L_{n}$ sets is a compact $L_{n}$ set.

Proof. The proof of part (a) is obvious and the proof of part (b) is contained in the proof of completeness of $\mathcal{K}$ referred to above. We turn therefore to part (c). Let $d\left(S^{k}, S\right)=\epsilon_{k}$ and let $p$ and $q$ be points of $S$. Then for each $k, p$ and $q$ are in $S_{\left(\epsilon_{k}\right)}^{k}$. By Theorem $1, S_{\left(\epsilon_{k}\right)}^{k}$ is an $L_{n}$ set. Thus there exist points $p_{1}^{k}, p_{2}^{k}, \cdots, p_{n-1}^{k}$ in $S_{\left(e_{k}\right)}^{k}$ such that the $n$-line $\left\langle p, p_{1}^{k}, p_{2}^{k}, \cdots, p_{n-1}^{k}, q\right\rangle$ is contained in $S_{\left(\epsilon_{k}\right)}^{k}$. Since $S_{\left(e_{k}\right)}^{k} \subset S_{\left(2 \epsilon_{k}\right)}$ we have $\left\langle p, p_{1}^{k}, \cdots, p_{n-1}^{k}, q\right\rangle \subset S_{\left(2 \epsilon_{k}\right)}$. We choose a subsequence of $\left\{S^{k}\right\}$ for which the corresponding subsequences $\left\{p_{1}^{k}\right\}, \cdots,\left\{p_{n-1}^{k}\right\}$ converge to the points $p_{1}, \cdots, p_{n-1}$. Since $S$ is closed and $\epsilon_{k} \rightarrow 0$, the $n$-line $\left\langle p, p_{1}, \cdots, p_{n-1}, q\right\rangle$ lies in $S$.

3. $L_{n}$ sets and connectedness. We are now ready to characterize compact, connected sets in terms of compact $L_{n}$ sets. We begin with three lemmas.

LEMMA 1. If $S$ is a compact, connected set and $\epsilon>0$ then there exists a positive integer $n$ such that $S_{(\epsilon)}$ is an $L_{n}$ set.

Proof. The collection of open $\epsilon$-discs about points of $S$ forms a cover of $S$ from which a finite subcover $D_{1}, \cdots, D_{N}$ can be selected. Consider the network formed by the line segments joining the centers of each pair $D_{i}$ and $D_{j}$ of overlapping discs. The centers of any two of the $N$ discs are joined by an $(N-1)$-line of this network. It follows that any two points of $S$ can be joined by an $(N+1)$-line in $\bigcup_{i=1}^{N} D_{i}$, hence any two points of $S_{(\epsilon)}$ can be joined by an $(N+3)$-line in $S_{(e) \text {. }}$

Lemma 2. The limit $S$ of a sequence $\left\{S^{k}\right\}$ of compact, connected sets is compact and connected.

Proof. Using the fact that $\left\{S^{k}\right\}$ is a Cauchy sequence we can select a subsequence of $\left\{S^{k}\right\}$ and parallel bodies of the members of this subsequence, such that these parallel bodies form a decreasing sequence of sets approaching $S$. By Lemma 1, these parallel bodies are connected. The conclusion of the lemma follows from Theorem $2(\mathrm{~b})$ and the fact that the intersection of a decreasing sequence of compact, connected sets is compact and connected. 
LEMMA 3. Any compact, connected set $S$ is the limit of a sequence of compact $L_{n}$ sets.

The proof follows immediately from Lemma 1 and Theorem 2(a). The following theorem is an immediate consequence of Lemma 2 and Lemma 3.

TheOREM 3. A necessary and sufficient condition that the set $S$ be compact and connected is that $S$ be the limit of a sequence of compact $L_{n}$ sets.

\section{REFERENCES}

1. Alfred Horn and F. A. Valentine, Some properties of $L_{n}$ sets in the plane, Duke Math. J. 16 (1949), 131-140.

2. A. M. Macbeath, Compactness theorems, Seminar on Convex Sets, 1949-1950, Chapter V, pp. 41-51, The Institute for Advanced Study.

University of California, Santa Barbara and

Santa Barbara, California 